\title{
Path Following Control for a Class of Electro-Mechanical Systems and Its Application
}

\author{
Mitsuru Taniguchi ${ }^{1, a}$ and Kenji Fujimoto ${ }^{1, b}$ \\ ${ }^{1}$ Dept. Mechanical Science and Engineering, Graduate School of Engineering \\ Nagoya University, Furo-cho, Nagoya, 464-8603, Japan \\ ataniguchi.mitsuru@h.nagoya-u.jp, bfujimoto@nagoya-u.jp
}

Keywords: Electro-mechanical systems, feedback control, path following control

\begin{abstract}
This paper is devoted to path following control for electro-mechanical systems described by the port-Hamiltonian form. Path following control is investigated mainly for mechanical systems since the desired path is characterized by its 'position'. Therefore, most of the existing results use the nature of second order differential equations since mechanical systems are described by them. The present paper proposes a new path following controller for 3rd order differential equations described by the port-Hamiltonian form. This is done by generalizing the authors' former result on passive velocity field control for mechanical systems.
\end{abstract}

\section{Introduction}

Path following control, whose objective is to make the state of the system track the desired path, is an important task for control of mechanical systems. This control task is investigated mainly for mechanical systems for which 'position' plays an important role since the desired path is characterized by its 'position.' Therefore, most of the existing results use the nature of second order differential equations since mechanical systems are described by them. The purpose of the present paper is to provide a new class of path following controllers for electro-mechanical systems which are described by 3rd (or higher) order differential equations.

Most of the existing results for this problem use the distance between the current state (position) and its desired path. See, e.g., [1,2]. However, it is difficult to measure the smallest distance between the current state and the desired path for complicated desired paths. Therefore several methods not using the distance are proposed to overcome this problem. See e.g. $[3,4,5,6]$ and the references therein. The authors proposed path following controllers in $[7,8]$ by generalizing the result in [6]. We re-formulated the existing results to cope with port-Hamiltonian systems and derive a path following control method applicable to a wider class of systems. Most of the existing path following control methods are only applicable to mechanical systems.

The authors' former result in [7] solves the path following control problem by adopting a virtual potential function which takes its minimum value on the desired path. The vector field of the feedback system with respect to the generalized momentum is designed in such a way that the virtual potential function decreases smoothly. The generalized momentum is decomposed into two elements. One is the direction tangent to the desired path (i.e. it is perpendicular to the gradient of the potential function), and the other is orthogonal to the former. Path following control is achieved by controlling the latter element since the potential function depends only on it. Although this method works for mechanical systems, it is not applicable to electro-mechanical systems directly since the two elements of the generalized momentum can not be controlled independently.

In the present paper, we propose a path following control method for electro-mechanical systems based on [7]. This method employs the virtual potential function. For the mechanical part of the system, the generalized momentum is decomposed in a similar way to the authors' former result. For the electronic part, the state is decomposed into three elements: 'gradient,' 'velocity,' and the rest. The potential function depends only on the 'gradient' element. The velocity to follow the desired path depends only on the 'velocity' element. Path following control is achieved by controlling the first element, while the velocity to follow the desired path is assigned by controlling the second element. 


\section{Problem Setting}

Let us consider the following linear port-Hamiltonian system.

$$
\left(\begin{array}{c}
\dot{q} \\
\dot{p} \\
\dot{\varphi}
\end{array}\right)=\left(\begin{array}{ccc}
0 & I & 0 \\
-I & -D & K_{m} \\
0 & -K_{m} & -R
\end{array}\right)\left(\begin{array}{c}
\frac{\partial H^{\mathrm{T}}}{\partial q} \\
\frac{\partial H}{\partial p}^{\mathrm{T}} \\
\frac{\partial H}{\partial \varphi}^{\mathrm{T}}
\end{array}\right)+\left(\begin{array}{l}
0 \\
0 \\
G
\end{array}\right) u, \quad H=\underbrace{\frac{1}{2} \varphi^{\mathrm{T}} L^{-1} \varphi}_{E(\varphi)}+\underbrace{\frac{1}{2} p^{\mathrm{T}} M^{-1} p}_{K(p)}
$$

Here $(q, p, \varphi) \in \mathrm{R}^{l} \times \mathrm{R}^{l} \times \mathrm{R}^{l}$ is the state. $I$ is the identity matrix. $D=D^{\mathrm{T}} \geq 0 \in \mathrm{R}^{l \times l}$ describes the friction of the mechanical part. $R=R^{\mathrm{T}} \geq 0 \in \mathrm{R}^{l \times l}$ describes the resistance in the circuit. The function $E(\varphi) \in \mathrm{R}$ with $L=L^{\mathrm{T}}>0 \in \mathrm{R}$ describes the electric energy and $K(p) \in \mathrm{R}$ with $M=M^{\mathrm{T}}>0 \in \mathrm{R}^{l \times l}$ describes the kinetic energy. $H(p, \varphi) \in \mathrm{R}$ describes the total energy. The matrix $G$ is nonsingular. The state $q$ and $p$ denote the position and the momentum of the mechanical part, respectively. The state $\varphi$ denote that of the electronic part.

The objective of the present paper is to make the state $q$ track its desired path while to make the momentum $p$ tracks its desired value. In the authors' former results [7, 8], we proposed path following controllers with a very similar objective for the following port-Hamiltonian system.

$$
\left(\begin{array}{l}
\bar{q} \\
\bar{p}
\end{array}\right)=\underbrace{\left(\begin{array}{cc}
0 & \bar{J}_{12}(\bar{q}) \\
\bar{J}_{12}(\bar{q})^{\mathrm{T}} & \bar{J}_{22}(\bar{q}, \bar{p})
\end{array}\right)}_{\bar{J}(\bar{q}, \bar{p})}\left(\begin{array}{c}
\frac{\partial \bar{H}^{\mathrm{T}}}{\partial \bar{q}} \\
\frac{\partial \bar{H}^{\mathrm{T}}}{\partial \bar{p}}
\end{array}\right)+\left(\begin{array}{c}
0 \\
\bar{G}(\bar{q})
\end{array}\right) u
$$

The main idea adopted in the present paper is to regard $\bar{q}=\left(q^{\mathrm{T}}, p^{\mathrm{T}}\right)^{\mathrm{T}}, \bar{p}=\varphi$ and apply the idea of the path following control of the authors' former result. Please note that the plant system (1) taken in this paper does not have the form (2) since the left upper part of the matrix $\bar{J}(\bar{q}, \bar{p})$ is non-zero for the system (1). The following section will show how to overcome this problem and how to derive a path following controller for the electro-mechanical system (1). In this paper, the inner product and the norm are defined by

$$
\left\langle x_{1}, x_{2}\right\rangle_{A}:=x_{1}^{\mathrm{T}} A x_{2} \text { and }\left\|x_{1}\right\|_{A}:=\left\langle x_{1}, x_{1}\right\rangle_{A}^{1 / 2} \text {. }
$$

\section{Main Result}

This section gives themain result of the present paper path following control of the port-Hamiltonian system (1) based on the authors' former result in [7]. Here we assume that the desired path is characterized by the configuration state $q$. A potential function of $q$ is designed in such a way that it takes its minimum value on the desired path and the momentum $p$ is decomposed into two elements in a similar way to the former result.

Unlike the result [7], the state has electronic part $\varphi$ which is decomposed into three elements. Let us consider a potential function $U(q) \in \mathrm{R}$ which takes its minimum value on the desired path. More precisely, the potential function $U(q)$ is chosen in such a way that the following assumption holds.

Assumption $1 U(q) \geq 0$ and it takes its minimum value 0 if and only if $q$ is on the desired path. We call the value of this potential function $U(q)$ potential energy. Furthermore, let us define the 'velocity' vector $p_{w}(q)$ on the phase space.

Assumption 2 The vector valued function $p_{w}(q) \in R^{l}$ satisfies the following conditions.

(i) $P w(q) \neq 0$ if $\mathrm{q}$ is on the desired path.

(ii) $\left\langle(\partial U / \partial q)^{T}, p_{w}(q)\right\rangle_{M^{-1}}=0$.

(iii) The measure of the set $\left\{q \mid p_{w}(q)=0\right\}$ is 0 . 
(iv) If $p \neq 0, p_{w} \neq 0$ and there exists $k(t) \in \mathrm{R}$ such that $p(t)=k(t) p_{w}(t)$ for all time $t$, then $(\partial U / \partial q)^{T}(q(t)) \equiv 0, \forall t \geq 0$ holds only if $q(t)$ is on the desired path for $\forall t \geq 0$.

In what follows, we consider a region close to the desired path on which $p_{w}(q) \neq 0$ holds and semi-global stability of the desired path within this region is discussed. The function $p_{w}(q)$ dose not always exist for all systems and the desired paths. For example, if the desired path crosses itself $p_{w}(q)$ dose not exist. Let $p_{w e}(q)$ denotes the normalized version of $p_{w}(q)$, that is, $p_{w e}(q)$ $:=p_{w}(q) /\left\|p_{w}(q)\right\|_{M^{-1}}$. Due to Assumptions 1 and 2, if the state is on the desired path at $t=0$, and if $p$ is parallel to $p_{w e}(q)$, i.e., there exists a scalar $k(t)$ satisfying $p(t)=k(t) p_{w e}(q(t))$ for $\forall t \geq 0$, then the state will stay on the desired path for all $t \geq 0$.

Next let us decompose $p$ into two elements: one is linearly dependent on $p_{w e}(q)$ and the other is orthogonal to $p_{w e}(q)$ which is denoted by $p_{\tilde{w}}(q, p)$. That is, $p$ is decomposed as

$$
p=\alpha_{p}(q, p) p_{w e}(q)+p_{\tilde{w}}(q, p),\left\langle p_{w e}(q), p_{\tilde{w}}(q, p)\right\rangle_{M^{-1}}=0
$$

where $p(q, p):=\left\langle p_{w e}(q), p\right\rangle_{M^{-1}}$ and $p_{\tilde{w}}(q, p):=p-\alpha_{p}(q, p) p_{w e}(q)$.

According to the decomposition (3), we can decompose the kinetic energy as

$$
K(p)=K_{w}(q, p)+K_{\tilde{w}}(q, p), K_{w}(q, p)=\frac{1}{2} \alpha_{p}(q, p)^{2}, K_{\tilde{w}}(q, p)=\frac{1}{2}\left\langle p_{\tilde{w}}(q, p), p_{\tilde{w}}(q, p)\right\rangle_{M^{-1}} .
$$

Here $K_{w}(q, p)$ and $K_{\tilde{w}}(q, p)$ denote the kinetic energy with respect to the desired direction $p_{w e}(q)$ and that with respect to the undesired one $p_{\tilde{w}}(q, p)$.

As stated above, Assumptions 1 and 2 suggest that path following control is achieved if the potential energy $U(q)$ takes its minimum value 0 and if the phase state $p$ is parallel to $p_{w e}(q)$. This means that the path following control is achieved if $K_{\tilde{w}}(q, p)+U(q)$ takes its minimum value 0 . The velocity to follow the desired path is a function of $q$ and $\alpha_{p}(q, p)$, since $\dot{q}=\alpha_{p}(q, p) M^{-1} p_{w e}(q)$ if $K_{\tilde{w}}$ $(q, p)+U(q)=0$. The time derivative of $K_{\tilde{w}}(q, p)+U(q)$ and $\alpha_{p}(q, p)$ along the port-Hamiltonian system (1) is calculated as

$$
\begin{aligned}
\frac{\mathrm{d}}{\mathrm{d} t}\left(U+K_{\tilde{w}}\right) & =\left\langle\frac{\partial U^{\mathrm{T}}}{\partial q}+K_{m} L^{-1} \varphi-D M^{-1} p-\alpha_{p} \frac{\partial p_{w e}}{\partial q} M^{-1} p, p_{\tilde{w}}\right\rangle_{M^{-1}} \\
\frac{\mathrm{d} \alpha_{p}}{\mathrm{~d} t} & =\left\langle K_{m} L^{-1} \varphi-D M^{-1} p, p_{w e}\right\rangle_{M^{-1}}+\left\langle\frac{\partial p_{w e}}{\partial q} M^{-1} p, p\right\rangle_{M^{-1}}
\end{aligned}
$$

Let us define the vector $\varphi_{w}(q):=L K^{-1} p_{w e}(q)$ and $\varphi_{\hat{w}}(q, p):=L K^{-1} p_{\tilde{w}}(q, p)$. By this definition,

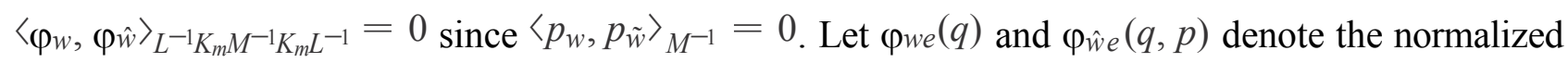
versions of $\varphi_{w}(q)$ and $\varphi_{\hat{w}}(q, p)$, that is, $\varphi_{w e}(q):=\varphi_{w}(q) /\left\|_{\varphi_{w}}(q)\right\|_{L^{-1} K_{m} M^{-1} K_{m} L^{-1}}=\varphi_{w}(q)$ and $\varphi_{\hat{w} e}(q$,

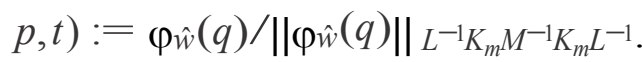

When $p_{\tilde{w}}(t)=0$, define $\varphi_{\hat{w} e}(q, p, t)=\lim _{\Delta t \rightarrow 0 \varphi \hat{w} e}(q, p, t-\Delta t)$. For continuity of $\varphi_{\hat{w} e}$, let us adopt the following assumption.

Assumption 3 There exists an integer $i \geq 0$ such that $\mathrm{d}^{i} p_{\tilde{w}}(q, p) / \mathrm{d} t^{i} \neq 0$ for all time $t$. This Assumption means that $(q, p) \in\left\{(q, p) \mid p_{\tilde{w}}(q, p)=0\right\}$ is not an invariant set for the closed loop system. Therefore $\varphi_{\hat{w}}$ is continuous in $t$. In what follows, we denotes $B:=L^{-1} K_{m} M^{-1} K_{m} L^{-1}$ for simplicity.

Now $\varphi$ is decomposed as

$$
\varphi=\alpha_{\varphi w}(q, \varphi) \varphi_{w e}(q)+\alpha_{\varphi \hat{w}}(q, p, \varphi, t) \varphi_{\hat{w} e}(q, p, t)+\varphi_{\tilde{w}}(q, p, \varphi, t)
$$


with $\left\langle\varphi_{w e}, \varphi_{\tilde{w}}\right\rangle_{B}=\left\langle\varphi_{\hat{w} e}, \varphi_{\tilde{w}}\right\rangle_{B}=0$ where $\varphi_{w}(q, \varphi):=\left\langle\varphi_{w e}(q), \varphi\right\rangle_{B}, \varphi_{\hat{w}}(q, p, \varphi, t):=\left\langle\varphi_{\hat{w} e}(q, p, t), \varphi\right.$ \rangle$_{B}$ and $\varphi_{\tilde{w}}(q, p, \varphi, t):=\varphi^{-} \alpha_{\varphi_{w}}(q, \varphi) \varphi_{w e}(q)-\alpha \varphi_{\hat{w}}(q, p, \varphi, t) \varphi_{\hat{w} e}(q, p, t)$. That is $\varphi$ is decomposed into three elements: one is the element which is linearly dependent on $\varphi_{w e}(q)$, another one is the element which is linearly dependent on $\varphi_{\hat{w} e}(q, p, t)$ and the other is orthogonal to them which is denoted by $\varphi_{\tilde{w}}(q, p, \varphi)$. From Equations (4) and (6), $\mathrm{d}\left(U+K_{\tilde{w}}\right) / \mathrm{d} t$ is calculated as

$$
\frac{\mathrm{d}}{\mathrm{d} t}\left(U+K_{\tilde{w}}\right)=\alpha_{\varphi \hat{w}}\left\|p_{\tilde{w}}\right\|_{M^{-1}}+\left\langle\frac{\partial U^{\mathrm{T}}}{\partial q}-D M^{-1} p-\alpha_{p} \frac{\partial p_{w e}}{\partial q} M^{-1} p, p_{\tilde{w}}\right\rangle_{M^{-1}} .
$$

Therefore we can let the state of the system converge to the desired path by selecting $\alpha \varphi \hat{w}$ in such

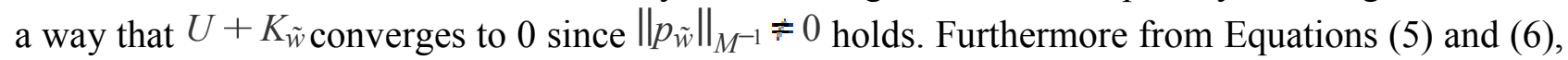
$\mathrm{d} \alpha_{p} / \mathrm{d} t$ is calculated as

$$
\frac{\mathrm{d} \alpha_{p}}{\mathrm{~d} t}=\alpha_{\varphi w}-\left\langle D M^{-1} p, p_{w e}\right\rangle_{M^{-1}}+\left\langle\frac{\partial p_{w e}}{\partial q} M^{-1} p, p\right\rangle_{M^{-1}} .
$$

Therefore we can assign the velocity to follow the desired path by selecting $\alpha \varphi w$ in such a way that $\alpha p$ converges to its desired value.

In the sequel, a path following controller is designed by four steps. In the first step, a controller called nominal controller is applied to make $\alpha_{\varphi w}, \alpha_{\varphi_{\hat{w}}}$ and $\left\|_{\varphi_{\tilde{w}}}\right\|_{B}$ constant in order to control them independently in Section 3.1. In the second step, another controller called asymptotic controller is added to make $\varphi_{\tilde{w}}(q, p, \varphi, t)$ converge to 0 in Section 3.2. In the third step, a controller called velocity controller is added to assign the desired velocity in Section 3.3. In the forth step, a controller called tracking controller is added to make the $U(q)$ and $K_{\tilde{w}}(q, p)$ converge to 0 in order that state stays on the desired path in Section 3.4.

\subsection{Nominal controller}

This subsection gives the nominal controller whose objective is to make $\alpha \varphi w, \alpha \varphi_{\hat{w}}$ and $\left\|_{\varphi_{\hat{w}}}\right\|_{B}$ constant in order to control them independently.

Theorem 1 Consider the port-Hamiltonian system (1) with a vector $p w(q)$. Suppose that Assumption 2 and Assumption 3 hold. Then $\alpha \varphi w, \alpha_{\varphi \hat{w}}$ and $\left\|_{\varphi_{\tilde{w}}}\right\|_{B}$ are constant along the path of the closed loop system derived by the nominal controller defined by

$$
\begin{aligned}
u_{n}= & G^{-1} K_{m} M^{-1} p+G^{-1} R L^{-1} \varphi+\alpha_{\varphi w} G^{-1} \frac{\mathrm{d} \varphi_{w e}}{\mathrm{~d} t}+\alpha_{\varphi \hat{w}} G^{-1} \frac{\mathrm{d} \varphi_{\hat{w} e}}{\mathrm{~d} t} \\
& \left.-\alpha_{\varphi \hat{w}}\left(\frac{\mathrm{d} \varphi_{\hat{w} e}}{\mathrm{~d} t}, \varphi_{w e}\right\rangle_{B} G^{-1} \varphi_{w e}-\left\langle\frac{\mathrm{d} \varphi_{w e}}{\mathrm{~d} t}, \varphi\right\rangle_{B} G^{-1} \varphi_{w e}-\alpha_{\varphi w} \frac{\mathrm{d} \varphi_{w e}}{\mathrm{~d} t}, \varphi_{\hat{w} e}\right)_{B} G^{-1} \varphi_{\hat{w} e}-\left\langle\frac{\mathrm{d} \varphi_{\hat{w} e}}{\mathrm{~d} t}, \varphi\right\rangle_{B} G^{-1} \varphi_{\hat{w} e} .
\end{aligned}
$$

Here $\mathrm{d} \varphi$ wel $\mathrm{d} t$ and $\mathrm{d} \varphi_{\hat{w} e} / \mathrm{d} t$ are time derivatives of $\varphi_{w e}$ and $\varphi_{\hat{w} e}$ along the port-Hamiltonian system (1) calculated as

$$
\begin{aligned}
\frac{\mathrm{d} \varphi_{w e}}{\mathrm{~d} t}= & \frac{\partial \varphi_{w e}}{\partial q} M^{-1} p \\
\frac{\mathrm{d} \varphi_{\hat{w} e}}{\mathrm{~d} t}= & \frac{1}{\left\|p_{\tilde{w}}\right\|_{M^{-1}}}\left\{-D L M^{-1} p+K_{m} \varphi-\left\langle-D M^{-1} p+K_{m} L^{-1} \varphi, p_{w e}\right\rangle_{M^{-1}} L p_{w e}-\alpha_{p} L \frac{\partial p_{w e}}{\partial q} M^{-1} p\right. \\
& -\left(\left\langle-D M^{-1} p+K_{m} L^{-1} \varphi, \frac{p_{\tilde{w}}}{\left\|p_{\tilde{w}}\right\|_{M^{-1}}}\right\rangle_{M^{-1}}-\alpha_{p}\left\langle L \frac{\partial p_{w e}}{\partial q} M^{-1} p, \frac{p_{\tilde{w}}}{\left\|p_{\tilde{w}}\right\|_{M^{-1}}} \lambda_{M^{-1}}\right) L \frac{p_{\tilde{w}}}{\left\|p_{\tilde{w}}\right\|_{M^{-1}}}\right\} .
\end{aligned}
$$

Let us consider a feedback system with the port-Hamiltonian system (1) with the feedback $u=u_{n}$ $+\bar{u}$. The time derivatives of $\alpha \varphi w, \alpha \varphi w^{\wedge}$ and $\left\|\varphi_{\varphi_{\tilde{w}}}\right\|_{B}$ along the closed loop system are calculated as

$$
\frac{\mathrm{d} \alpha_{\varphi_{w}}}{\mathrm{~d} t}=\left\langle G \bar{u}, \varphi_{w e}\right\rangle_{B}, \frac{\mathrm{d} \alpha_{\varphi \hat{w}}}{\mathrm{~d} t}=\left\langle G \bar{u}, \varphi_{\hat{w} \varepsilon}\right\rangle_{B}, \frac{\mathrm{d}\left\|\varphi_{\tilde{w}}\right\|_{B}}{\mathrm{~d} t}=\left\langle G \bar{u}, \frac{\varphi_{\tilde{w}}}{\left\|\varphi_{\tilde{w}}\right\|_{B}}\right\rangle_{B} .
$$




\subsection{Asymptotic controller}

Next let us introduce the asymptotic controller whose objective is to reduce $\left\|_{\varphi_{\tilde{w}}}\right\|_{B}$.

Theorem 2 Consider the port-Hamiltonian system (1) with a vector $p_{w}(q)$. Suppose that Assumption 2 holds and define the asymptotic controller by

$$
u_{a}=-\beta_{a} G^{-1} \varphi_{\tilde{w}}
$$

where a continuous function $\beta a(q, p, \varphi, t)>0 \in \mathrm{R}$ is a design parameter. Then $\left\|_{\varphi_{\tilde{w}}}\right\|_{B}$ monotonically decreases and converges to 0 as $t \rightarrow \infty$ along the state trajectory of the closed loop system with the feedback $u=u_{n}+u_{a}$.

\subsection{Velocity Control}

This subsection gives the velocity controller to assign the velocity to follow the desired path. The velocity to follow the desired path is a function of $q$ and $\alpha_{p}(q, p)$, since ${ }^{*} q=\alpha_{p}(q, p) M^{-1} p_{w e}(q)$ when the system follows the desired path. Therefore the objective of velocity controller is to make $\alpha_{p}(q, p)$ converge to its desired value $\alpha_{p r}(q, t)$.

Let us calculate the time derivative of $\alpha_{p}-\alpha_{p r}$ along the state trajectory of the system (1) as

$$
\frac{\mathrm{d}}{\mathrm{d} t}\left(\alpha_{p}-\alpha_{p r}\right)=\alpha_{\varphi w}-\left\langle D M^{-1} p, p_{w e}\right\rangle_{M^{-1}}+\left\langle\frac{\partial p_{w e}}{\partial q} M^{-1} p, p\right\rangle_{M^{-1}}-\frac{\mathrm{d} \alpha_{p r}}{\mathrm{~d} t} .
$$

This equation follows from Equation (5). $\mathrm{d}\left(\alpha_{p}-\alpha_{p r}\right) / \mathrm{d} t$ depends on $\alpha \varphi w$ bud dose not depend on $\alpha_{\varphi \hat{w}}$ and $\varphi_{\tilde{w}}$. Therefore we define $\alpha_{\varphi w r}(q, p, t)$ which is the desired value of $\alpha_{\varphi w}$ as

$$
\alpha_{\varphi w r}:=-\beta_{v 1}\left(\alpha_{p}-\alpha_{p r}\right)+\left\langle D M^{-1} p, p_{w e}\right\rangle_{M^{-1}}-\left\langle\frac{\partial p_{w e}}{\partial q} M^{-1} p, p\right\rangle_{M^{-1}}+\frac{\mathrm{d} \alpha_{p r}}{\mathrm{~d} t}
$$

where a continuous function $\beta_{v 1}(q, p, t)>0 \in \mathrm{R}$ is a design parameter. If $\alpha_{\varphi w}$ converge to its desired value $\alpha \varphi w r$, then

$$
\frac{\mathrm{d}}{\mathrm{d} t}\left\{\frac{1}{2}\left(\alpha_{p}-\alpha_{p r}\right)^{2}\right\}=-\beta_{v 1}\left(\alpha_{p}-\alpha_{p r}\right)^{2} \leq 0
$$

which says that $\alpha p$ converges to $\alpha p r$. Hence the velocity controller makes $\alpha \varphi w$ converge to $\alpha \varphi w r$.

Theorem 3 Consider the port-Hamiltonian system (1) with a scalar function $U(q)$, a continuously differentiable scalar function $\alpha p r(q, t)$ and a vector $p_{w}(q)$. Suppose that Assumptions 1 and 2 hold and define the velocity controller by

$$
u_{v}=-\beta_{v 2}\left(\alpha_{\varphi w}-\alpha_{\varphi w r}\right) G^{-1} \varphi_{w e}-\left(\alpha_{p}-\alpha_{p r}\right) G^{-1} \varphi_{w e}+\frac{\mathrm{d} \alpha_{\varphi w r}}{\mathrm{~d} t} G^{-1} \varphi_{w e}
$$

where a continuous function $\beta_{v 2}(q, p, t)>0 \in \mathrm{R}$ is a design parameter. Then $\alpha_{p}$ converges to $\alpha_{p r}$ as $t$ $\rightarrow \infty$ along the state trajectory of the closed loop system with the feedback $u=u_{n}+u_{a}+u_{v}$.

\subsection{Path following control}

This subsection gives the tracking controller to make the system converge to the desired path. The system tracks the desired path when $U+K_{\tilde{w}}=0$.

Let us calculate the time derivative of $U+K_{\tilde{w}}$ along the state trajectory of the system (1) as

$$
\frac{\mathrm{d}}{\mathrm{d} t}\left(U+K_{\tilde{w}}\right)=\alpha_{\varphi \hat{w}}\left\|p_{\tilde{w}}\right\|_{M^{-1}}+\left\langle\frac{\partial U^{\mathrm{T}}}{\partial q}-D M^{-1} p-\alpha_{p} \frac{\partial p_{w e}}{\partial q} M^{-1} p, p_{\tilde{w}}\right\rangle_{M^{-1}} .
$$

$\mathrm{d}\left(U+K_{\tilde{w}}\right) / \mathrm{d} t$ depends on $\alpha \varphi_{\hat{w}}$ bud dose not depend on $\alpha \varphi w$ and $\varphi_{\tilde{w}}$. Therefore we define $\alpha \varphi_{\hat{w} r}(q, p, t)$ which is the desired value of $\alpha \varphi \hat{w}$ as

$$
\alpha_{\varphi \hat{w} r}:=-\left(\beta_{t 1}\left\|p_{\tilde{w}}\right\|_{M^{-1}}+\left\langle\frac{\partial U^{\mathrm{T}}}{\partial q}-D M^{-1} p-\alpha_{p} \frac{\partial p_{w e}}{\partial q} M^{-1} p, \frac{p_{\tilde{w}}}{\left\|p_{\tilde{w}}\right\|_{M^{-1}}}\right\rangle_{M^{-1}}\right) \text {. }
$$


where a continuous function $\beta_{t 1}(q, p, t)>0 \in \mathrm{R}$ is a design parameter. If $\alpha \varphi w$ converges to its desired value $\alpha_{\varphi w r}$, then

$$
\frac{\mathrm{d}}{\mathrm{d} t}\left(U+K_{\tilde{w}}\right)=-\beta_{t 1}\left\langle p_{\tilde{w}}, p_{\tilde{w}}\right\rangle_{M^{-1}} \leq 0
$$

which says that $U+K_{\tilde{w}}$ reduces. Hence the tracking controller makes $\alpha_{\varphi \hat{w}}$ converge to $\alpha_{\varphi \hat{w}} r$.

Theorem 4 Consider the port-Hamiltonian system (1) with a scalar function U(q), a continuously differentiable scalar function $\alpha_{p r}(q, t)$ and a vector $p_{w}(q)$. Suppose that Assumptions 1 and 2 hold and $\alpha_{p r}(q, t) \neq 0$ for $q$ satisfying $(\partial U / \partial q)(q)=0$. Define the tracking controller by

$$
u_{t}=-\beta_{t 2}\left(\alpha_{\varphi \hat{w}}-\alpha_{\varphi \hat{w} r}\right) G^{-1} \varphi_{\hat{w} e}-\left\|p_{\tilde{w}}\right\|_{M^{-1}} G^{-1} \varphi_{\hat{w} e}+\frac{\mathrm{d} \alpha_{\hat{\varphi} \hat{w} r}}{\mathrm{~d} t} G^{-1} \varphi_{\hat{w} e}
$$

where a continuous function $\beta_{t 2}(q, p, t)>0 \in \mathrm{R}$ is a design parameter. Then $\alpha_{p}$ converges to $\alpha_{p r}$ as $t$ $\rightarrow \infty$ and the state converges to the desired path along the state trajectory of the closed loop system with the feedback $u=u_{n}+u_{a}+u_{v}+u_{t}$ if Assumption 3 holds.

\section{Conclusions}

This paper is devoted to path following control for a class of linear electro-mechanical systems. We decompose the state of the electronic part of the system into three elements in such a way that the conventional path following control method can apply. Then we can obtain a path following controller by which the position tracks the desired path while its momentum tracks its desired value as well. Although the proposed method is for a linear electro-mechanical system, it provides a new class of controllers for 3rd order differential equations which are not reported in the literature. The future work would consider the nonlinear versions of the proposed method.

\section{References}

[1] M. Sampei, T. Tamura, T. Kobayashi, and N. Shibui, "Arbitrary path tracking control of articulated vehicles using nonlinear control theory," IEEE Transaction on Control Systems Technology, 1995.

[2] H. C. Ho, J. Y. Yen, and S. S. Lu, "A decoupled path-following control algorithm based upon the decomposed trajectory error," International Journal of Machine Tolls \& Manufacture, vol. 39, no. 10 , pp. 1619-1630, 1999.

[3] J. K. Salisbury, "Active stiffness control of a manipulator in Cartesian coordinates," in Proc. 19th IEEE Conf. on Decision and Control, 1980.

[4] N. Hogan, "Impedance contro: An approach to manipulation," Trans. ASME, J. Dyn. Syst., Meas., Control, vol. 107, no. 1, pp. 1-24, 1985.

[5] P. Y. Li and R. Horowitz, "Passive velocity field control of mechanical manipulators," IEEE Trans. Robotics and Automation, vol. 15, no. 4, pp. 751-763, 1999.

[6] V. Duindam and S. Stramigioli, "Passive asymptotic curve tracking," in Proc. IFAC Workshop on Lagrangian and Hamiltonian Methods for Nonlinear Control, 2003, pp. 229-234.

[7] M. Taniguchi and K. Fujimoto, "Passive trajectory control for port-hamiltonian systems," in Proc. European Control Conference 2009, 2009, pp. 236-241.

[8] M. Taniguchi and K. Fujimoto, "Time-varying path following control for port-hamiltonian systems," in Proc. 48th IEEE Conf. on Decision and Control, 2009, pp. 3323-3328. 